\title{
Effect of the Surface Energy Barrier on Sorbate Diffusion in $\mathrm{AlPO}_{4}-5$
}

\author{
Gaurav Arya, Edward J. Maginn,* and Hsueh-Chia Chang \\ Department of Chemical Engineering, University of Notre Dame, Notre Dame, Indiana 46556
}

Received: September 18, 2000; In Final Form: December 19, 2000

\begin{abstract}
The importance of pore exit effects on the diffusion of molecules in $\mathrm{AlPO}_{4}-5$ pores is evaluated using two molecular modeling techniques. In the first approach, a dual control volume grand canonical molecular dynamics technique is used to obtain molecular fluxes of methane out of the truncated crystal as a function of temperature and sorbate loading. The simulation results indicate the presence of a low-temperature surface barrier for diffusion, which retards the flux of methane relative to its apparent flux in the intracrystalline regions of the material. This pore exit barrier tends to diminish as temperature and loading increase. An explanation based on clustering phenomena is proposed to explain the latter. Next, a simple activated transport model is proposed to predict the relative importance of the surface barrier on the transport of sorbates in $\mathrm{AlPO}_{4}-5$. The potential of mean force for a single sorbate molecule along the pore axis of a truncated crystal provides the required activation energy barriers for the model. The model correctly predicts the reduction in the importance of exit effects with an increase in the temperature. It is also observed that exit effects become more important as the ratio of the size of the sorbate molecule to the pore size approaches unity. In particular, exit effects are significant in micrometer-thick $\mathrm{AlPO}_{4}-5$ crystals in the case of large molecules such as $\mathrm{SnBr}_{4}$ and $\mathrm{CCl}_{4}$ at room temperature.
\end{abstract}

\section{Introduction}

Understanding the diffusion of molecules into, through, and out of zeolite pores is of crucial importance in catalytic and separation applications. For example, permeation through zeolite membranes consisting of polycrystalline films deposited on a support depends critically on both intra- and intercrystalline diffusion processes. The large number of crystal intergrowths, and the tortuous diffusion pathways that molecules follow to permeate the membrane, greatly complicates analysis. A similar process occurs in zeolite catalysts, which typically consist of a crystalline powder mixed with a binder and pressed into a pellet. Diffusion to and from the active sites involves a process in which molecules must enter the zeolite pores, diffuse within the pores to an active site, diffuse away from the active site, and eventually leave the pore. It is generally assumed that the major resistance in this process is the intracrystalline step, and that diffusion into and out of the pores is facile. This is not always the case, however, as certain zeolites can be tailored to have large surface barriers which contribute to their selectivity. ${ }^{1,2}$

The issue of the relative importance of intracrystalline and intercrystalline resistance to diffusion has also been proposed as a way of explaining the well-known discrepancy between so-called "microscopic" and "macroscopic" diffusion measurements. ${ }^{3}$ Microscopic techniques such as pulsed field gradient nuclear magnetic resonance (PFG NMR) and quasi-elastic neutron scattering techniques measure intracrystalline selfdiffusivities, while macroscopic methods such as gravimetric uptake rate experiments measure a combination of intercrystalline and intracrystalline diffusion under the presence of a transient concentration gradient. For some systems, the macroscopic measurements yield diffusivities several orders of

* To whom correspondence should be addressed. Phone: (219) 631 5687. Fax: (219) 631-8366. E-mail: ed@ @d.edu. URL: http://www.nd.edu/ $\sim$ ed. magnitude lower than the microscopic measurements. It has been postulated that the presence of surface barriers at the pore mouths retard intercrystalline diffusion and thus could explain this discrepancy.

The exact nature of the surface barrier is unclear. A barrier may exist due to the presence of structural defects at the surface imparted during preparation, ${ }^{4,5}$ e.g., from deposition of impenetrable material at the outside of individual crystals ${ }^{6}$ or by changes in the crystal structure due to chemical reactions during the process of cation exchange and hydrothermal treatment. ${ }^{7}$ Kärger et al. ${ }^{5}$ carried out ${ }^{129} \mathrm{Xe}$ PFG NMR measurements to determine the surface permeabilities of $\mathrm{NaX}, \mathrm{NaCaA}$, and ZSM-5 crystals. It was observed that surface resistance was important in $\mathrm{NaCaA}$ and ZSM-5, though similar experiments with methane did not show any indication of the existence of diffusional barriers on the external surface.

There also exists a natural barrier at the pore mouths due to the discontinuity in the zeolite potential field at the surface..$^{8,9}$ Kočiřík et al. ${ }^{8}$ used a microdynamic model to show that for small crystals this natural surface resistance can exceed the intracrystalline diffusional resistance by several orders of magnitude. Barrer ${ }^{2}$ and later Kärger ${ }^{10}$ derived generalized flux expressions that accounted for the "evaporation barrier" that arises due to the potential energy discontinuity at the zeolite surface. It was shown that, under certain conditions, the presence of the pore exit can actually enhance the flux observed in a bed of zeolite crystals. ${ }^{10}$ There have been several molecular dynamics (MD) studies of the pore entrance problem also. VignéMaeder et al. ${ }^{11}$ used MD to examine the trajectories of argon and xenon atoms passing through the outer surface of MFI- and MOR-type zeolite crystals. They observed that the flux of the larger molecule, xenon, was significantly retarded relative to that of argon. This implies the presence of some form of barrier for pore entrance, the magnitude of which depends on the size of the sorbate molecule. This was a significant finding, since 


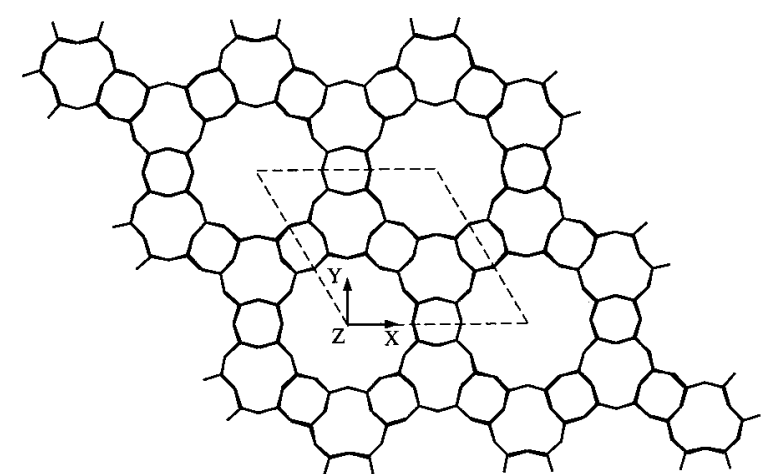

Figure 1. Schematic diagram of $\mathrm{AlPO}_{4}-5$ on the $x-y$ plane showing the pore running in the $z$ direction. The crystal unit cell is shown with dashed lines.

most MD studies have only considered intracrystalline diffusion and tend to agree quite well with the microscopic experimental techniques. ${ }^{12}$ Ford and Glandt ${ }^{9}$ used MD to obtain mass-transfer coefficients for hard spheres passing through a barrier comprised of slit pore mouths. They observed a significant mass-transfer resistance associated with the penetration of the pore mouth, which they attributed to steric and energetic interactions between the diffusing molecules and the atoms comprising the pore mouth. They also found that this resistance is extremely sensitive to slight changes in the hard sphere size whenever the ratio of the penetrating molecule diameter and the pore diameter is near unity. In a later study, Ford and Glandt ${ }^{13}$ concluded that adsorption resistance is also a significant source of mass-transfer resistance, especially at low temperature and when the interactions between the pore walls and the diffusing species are strong. Webb and Grest ${ }^{14}$ have carried out large-scale MD simulations to examine the mechanism by which alkanes enter the pores of zeolites. They found that the process whereby a chain molecule enters a zeolite pore is a complex sequence of events involving surface adsorption, surface diffusion, and finally pore entrance.

Most of the previous studies have focused on the natural energy barrier for adsorption (i.e., pore entrance). There may also exist a large energy (or "evaporation") barrier at the pore exit. . $^{2,10}$ This can be understood by considering the loss in favorable dispersion energy that a molecule gives up when it leaves a pore, where it is in intimate contact with the lattice atoms, and moves out into a low-pressure gas phase. The objective of the present study is to quantify the magnitude of this exit effect in $\mathrm{AlPO}_{4}-5$ for a range of sorbates at varying temperatures and loadings. $\mathrm{AlPO}_{4}-5$ is chosen as the model zeolite system because it has a simple pore structure consisting of nonintersecting and approximately cylindrical pores of nominal diameter $7.3 \AA$ running in the [001] direction. Figure 1 shows the pore structure of the zeolite in a schematic form. The unit cell of $\mathrm{AlPO}_{4}-5$ has lattice parameters $a=13.726 \AA$, $b=13.726 \AA, c=8.484 \AA, \alpha=90^{\circ}, \beta=90^{\circ}$, and $\gamma=120^{\circ}$. In this paper the $\mathrm{AlPO}_{4}-5$ crystal is assumed to be rigid and free of defects. First, dual control volume grand canonical molecular dynamics (DCV-GCMD) simulations will be conducted for methane in both a periodic and truncated pore system to compute intracrystalline and intercrystalline flux rates, respectively. Although methane has relatively high transport rates in $\mathrm{AlPO}_{4}-5$, the DCV-GCMD simulations are still quite demanding; species having a much lower intercrystalline or intracrystalline mobility than methane are difficult to simulate with reasonable computational resources. To circumvent this problem, a simple activated transport model based on a coarsegrained potential of mean force is developed. The model is tested against the DCV-GCMD methane results and shown to give (a)

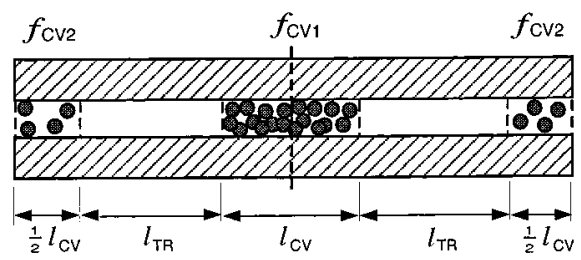

(b)

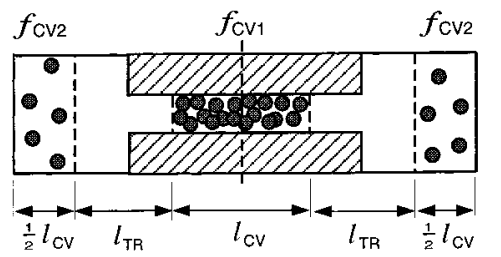

Figure 2. Schematic diagram of the setup of DCV-GCMD simulations to study (a) intracrystalline transport rates and (b) transport rates at the pore exit.

good agreement. The model is then used to make predictions as to the magnitude of the exit effect for the transport of other molecules in $\mathrm{AlPO}_{4}-5$.

\section{DCV-GCMD Simulations}

\subsection{Simulation Details.}

To understand the role of a pore exit on transport rates, the DCV-GCMD technique is used. ${ }^{15}$ In this approach, a constant chemical potential is maintained in two control volumes using a grand canonical Monte Carlo method (GCMC). The control volumes are kept at different chemical potentials and are separated by a diffusion or "transport" zone. The dynamic part of the technique involves a normal molecular dynamics simulation in the microcanonical ensemble. The steady-state flux that develops is calculated and then related to the chemical potential driving force through a transport coefficient.

For the study performed here, two different types of simulations are conducted. The first set of simulations are conducted to compute the intracrystalline transport coefficient. Figure $2 \mathrm{a}$ shows a two-dimensional schematic of the simulation cell used to compute intracrystalline fluxes. The second set of simulations is used to determine an effective transport coefficient associated with only the pore exit process. Figure $2 b$ shows the cell used to examine fluxes in the presence of a pore exit. As described later, these two transport coefficients will be used to determine the overall resistance to transport for a pore of arbitrary length. In both cases, a central control volume (CV1) within the zeolite pore is maintained at a high fugacity equal to $f_{\mathrm{CV} 1}$. The second control volume (CV2) is maintained at a lower fugacity, $f_{\mathrm{CV} 2}$. For the intracrystalline diffusion simulations, CV2 is located inside the zeolite pore, while, for the pore exit studies, CV2 is located outside the zeolite pore in the bulk phase. The length of the control volume is $l_{\mathrm{CV}}$, while the length of the transport zone is $l_{\mathrm{TR}}$.

For the intracrystalline simulations where both control volumes are inside the pore, periodic boundary conditions are implemented in the $z$ direction, and each control volume encompasses the entire cross-sectional area of the pore. A steady-state flux is produced in the two transport regions by maintaining a chemical potential difference between the control volumes. The length of the transport region is kept fixed at 42.42 $\AA$ (equivalent to five unit cell widths in the $z$ direction).

In the pore exit simulations, periodic boundary conditions are applied in all directions, because molecules in the bulk phase are free to cross boundaries in the $x$ and $y$ directions, as opposed to molecules constrained within the pores in the case of 
intracrystalline simulations. The transport region in the $z$ direction is positioned such that half of its length in the $z$ direction is inside the zeolite pore and the other half is in the bulk. The transport region should at least encompass the exit region, operationally defined here as twice the cutoff radius of the interaction potential $(20 \AA)$. For convenience, we chose a length of four unit cells, which is equivalent to $33.94 \AA$. This ensures that these simulations measure a fairly pure pore exit transport coefficient by including all the portions of the pore which are influenced by the pore mouth, and as little as possible the unaffected intracrystalline component of the pore. The lengths of the control volumes in both sets of simulations vary according to the sorbate loading desired.

The zeolite surfaces exposed to the bulk phase in the pore exit simulations are generated by cleaving the lattice at the edges of the unit cell. The surface on the "left" is created by cleaving the zeolite at the left edge of the unit cell, and therefore consists of tetravalent aluminum atoms at the surface, beneath which lies a layer of doubly coordinated oxygen atoms. The surface on the "right" side is produced by cleaving at the right edge of the unit cell and consists of singly coordinated oxygen atoms. There are other axial positions at which the zeolite may have been cleaved to produce other types of surfaces. As discussed below, however, the slight structural differences between the two surfaces used here have little impact on the pore exit flux. Thus, the choice of where to terminate the crystal does not appear to affect pore exit rates to a great extent. In addition, no attempt is made to accurately model the atomistic details of the surface for the following reason. The purpose of the present work is to assess the overall impact of the discontinuity in the sorbate-zeolite interactions on the diffusion process. While we chose a specific system (methane in $\mathrm{AlPO}_{4}-5$ ), we are not as interested in the specific details of this particular system as we are in the general trends. The exact chemical and physical nature of a zeolite surface is likely to be complex and certainly far from well understood. It is likely that the surface is terminated with hydroxyl groups, and may be slightly reconstructed. Trying to account for this detailed surface structure, however, would involve a degree of arbitrariness. Since it is probable that the detailed nature of the surface plays a secondary role in the diffusion process for a nonpolar species such as methane (unless of course the surface is deliberately altered to partially block the pores), we felt it was best to focus on an ideal pore system.

The control volumes are maintained at constant fugacity (and therefore constant chemical potential) by stochastic creation and deletion of sorbate molecules according to a fugacity explicit variation of the GCMC formalism. Molecules are inserted into the control volumes according to the insertion acceptance rule ${ }^{16}$

$$
\Pi_{m n}^{\mathrm{ins}}=\min \left(1, \frac{f \beta V}{N_{m}+1} \exp \left(-\beta \Delta v^{m n}\right)\right)
$$

and deleted according to the deletion acceptance rule

$$
\Pi_{n m}^{\mathrm{del}}=\min \left(1, \frac{N_{m}}{f \beta V} \exp \left(-\beta \Delta v^{n m}\right)\right)
$$

where $f$ is the fugacity of the fluid, $N_{m}$ is the number of molecules present in the control volumes in state $m, N_{n}=N_{m}$ +1 is the number of molecules in state $n, V$ is the volume of the control volumes, and $\Delta v$ is the difference in the potential energies of states $m$ and $n$. The molecules created are imparted velocities sampled from a Maxwell-Boltzmann distribution corresponding to the simulation temperature. The dynamic part of the simulation (molecular dynamics) uses a velocity-Verlet algorithm with a time step of 5 fs. After every 5 ps of the MD simulation, the GCMC routine is activated and a series of insertions and deletions (100 altogether) are performed in each of the control volumes. It is important to keep the frequency of insertions/deletions high enough to maintain a constant fugacity in the control volumes, but small enough so as not to alter the dynamics of molecules near the control volume-transport region interfaces. No thermostat is used in all the simulations as the temperature remained fairly constant without thermostating. This is because the control volumes become thermostated by the frequent insertions of molecules at the required temperature in the GCMC part of the simulations. In the case of pore exit simulations, there is a slight reduction in the temperature of molecules escaping from the pore potential well. The temperature of these molecules is gradually brought back to the preset temperature through collisions with molecules at the preset temperatures escaping from the end control volumes. We believe that this gradual temperature control is closer to what occurs in a real system, and so chose not to apply an external homogeneous thermostat.

The methane-methane interaction is modeled using a Lennard-Jones (LJ) potential with interaction parameters $\sigma=3.73$ $\AA$ and $\epsilon / k_{\mathrm{B}}=147.95 \mathrm{~K} .{ }^{17}$ The interactions between methane and the lattice are modeled by only considering interactions with the oxygen atoms of the zeolite. ${ }^{18}$ The methane-oxygen LJ parameters, also taken from ref 17 , are $\sigma=3.214 \AA$ and $\epsilon / k_{\mathrm{B}}$ $=133.3 \mathrm{~K}$. A cutoff radius $\left(r_{\mathrm{c}}\right)$ of $10 \AA$ is used in all calculations. To reduce computational effort, the sorbate-zeolite interaction potential is stored over a three-dimensional grid (this tabulation of potentials at each grid point is referred to as the potential map ${ }^{19}$ ) with roughly $0.2 \AA$ spacing, and an interpolating scheme is used during the simulations to rapidly compute the potential and forces on each sorbate arising from interactions with the lattice. We specifically require three potential maps: one for the periodic unit cell which does not "feel" the zeolitebulk interface, used in both intracrystalline and pore exit simulations, and the other two for the dissimilar exit regions on either side of the zeolite-bulk interface required for the pore exit simulations only.

To further reduce computational effort, interactions between sorbates in adjacent pores were neglected. Although it is possible for the separation between these molecules to be slightly less than the cutoff distance, these interactions typically account for less than $0.15 \%$ of the total potential energy a molecule experiences. In other words, the potential field is dominated by the zeolite and sorbates in the same pore, with neighboring pore sorbate-sorbate interactions being only a secondary effect which are not expected to significantly impact transport properties.

Both sets of DCV-GCMD simulations are conducted at three different average sorbate loadings: a low sorbate loading of 0.5 molecule/unit cell, a medium sorbate loading of 1.5 molecules/unit cell, and a high sorbate loading of 2.5 molecules/ unit cell. These are referred to as "L", "M", and "H", respectively, for the rest of the paper. In the intracrystalline simulations, the difference in the loadings between the middle control volume and the end control volumes is fixed at 0.25 molecule/unit cell. This means that the middle control volume is maintained at a loading 0.125 molecule/unit cell above the average and the end control volumes are maintained at a loading of 0.125 molecule/unit cell below the average. The fugacities required to maintain the control volumes at the desired loadings can be obtained from the adsorption isotherms. Figure 3 shows the computed adsorption isotherms for methane molecules in $\mathrm{AlPO}_{4}-5$ at five different temperatures ranging from 100 to 500 


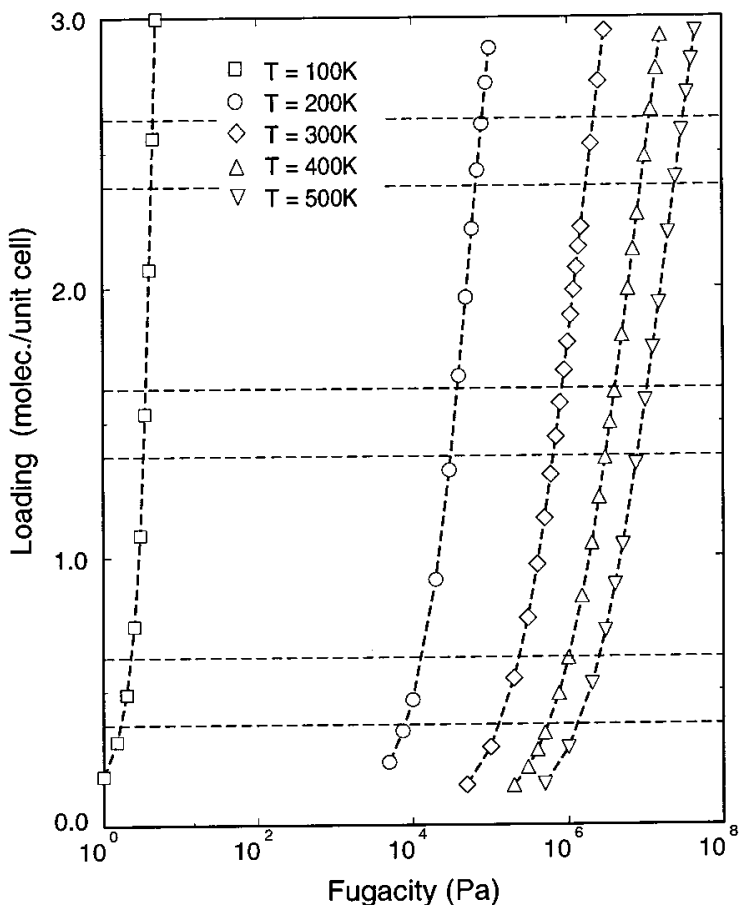

Figure 3. Adsorption isotherms for $\mathrm{CH}_{4}$ in $\mathrm{AlPO}_{4}-5$ at different temperatures. The dashed horizontal lines refer to the upper and lower loadings for the L, M, and $\mathrm{H}$ types of loadings. The symbols represent the values computed, while the dotted lines are to guide the eye.

TABLE 1: Fugacities Corresponding to the Required Loadings in the Two Control Volumes at Different Temperatures $^{a}$

\begin{tabular}{ccccrcc}
\hline \multirow{3}{*}{$T(\mathrm{~K})$} & $\begin{array}{c}\text { loading } \\
\text { level }\end{array}$ & $f_{\mathrm{CV} 1}(\mathrm{~Pa})$ & $f_{\mathrm{CV} 2}(\mathrm{~Pa})$ & $l_{\mathrm{CV}}{ }^{b}(\AA)$ & $t_{\mathrm{I}}(\mu \mathrm{s})$ & $t_{\mathrm{E}}(\mu \mathrm{s})$ \\
\hline 100 & $\mathrm{~L}$ & $2.27 \times 10^{0}$ & $1.67 \times 10^{0}$ & 339.36 & 2.0 & 2.0 \\
& $\mathrm{M}$ & $3.59 \times 10^{0}$ & $3.32 \times 10^{0}$ & 135.74 & 2.0 & 2.0 \\
& $\mathrm{H}$ & $4.58 \times 10^{0}$ & $4.31 \times 10^{0}$ & 67.87 & 2.0 & 2.0 \\
200 & $\mathrm{~L}$ & $1.35 \times 10^{4}$ & $8.00 \times 10^{3}$ & 339.36 & 1.0 & 1.0 \\
& $\mathrm{M}$ & $3.87 \times 10^{4}$ & $3.15 \times 10^{4}$ & 135.74 & 1.0 & 1.0 \\
& $\mathrm{H}$ & $8.12 \times 10^{4}$ & $6.73 \times 10^{4}$ & 67.87 & 1.0 & 1.0 \\
300 & $\mathrm{~L}$ & $2.35 \times 10^{5}$ & $1.33 \times 10^{5}$ & 339.36 & 1.0 & 1.0 \\
& $\mathrm{M}$ & $8.46 \times 10^{5}$ & $6.51 \times 10^{5}$ & 135.74 & 1.0 & 1.0 \\
400 & $\mathrm{H}$ & $2.20 \times 10^{6}$ & $1.75 \times 10^{6}$ & 67.87 & 1.0 & 1.0 \\
& $\mathrm{~L}$ & $1.01 \times 10^{6}$ & $5.55 \times 10^{5}$ & 339.36 & 0.5 & 0.5 \\
& $\mathrm{M}$ & $4.06 \times 10^{6}$ & $3.04 \times 10^{6}$ & 135.74 & 0.5 & 0.5 \\
& $\mathrm{H}$ & $1.16 \times 10^{7}$ & $8.97 \times 10^{6}$ & 67.87 & 0.5 & 0.5 \\
& $\mathrm{~L}$ & $2.51 \times 10^{6}$ & $1.37 \times 10^{6}$ & 339.36 & 0.5 & 0.5 \\
& $\mathrm{M}$ & $1.06 \times 10^{7}$ & $7.85 \times 10^{6}$ & 135.74 & 0.5 & 0.5 \\
& $\mathrm{H}$ & $3.17 \times 10^{7}$ & $2.43 \times 10^{7}$ & 67.87 & 0.5 & 0.5
\end{tabular}

${ }^{a}$ Also shown are the lengths of control volumes, $l_{\mathrm{CV}}$, and simulation lengths for the intracrystalline $\left(t_{\mathrm{I}}\right)$ and pore exit simulations $\left(t_{\mathrm{E}}\right) .{ }^{b}$ The length of CV2 is taken as the sum of the left and right end control volumes, and $l_{\mathrm{CV} 1}=l_{\mathrm{CV} 2}$ for all simulations.

$\mathrm{K}$. The dashed horizontal lines correspond to the desired sorbate loading of the central and end control volumes. The fugacities needed to maintain the required sorbate loadings in both the control volumes at the five different temperatures can be obtained from the intersections of the dashed lines with the adsorption isotherms. The values of these fugacities are tabulated in Table 1. The methane loadings considered here are well below the maximum attainable loading of methane in $\mathrm{AlPO}_{4}-5$, which is in excess of 7 molecules/unit cell. Typical loadings in experimental $\mathrm{AlPO}_{4}-5$ would correspond to equilibrium loadings at near atmospheric pressures and temperatures. For typical experimental pressures of $10^{5} \mathrm{~Pa}$ and temperatures of $300 \mathrm{~K}$, the equilibrium loading is about 0.3 molecule/unit cell (as seen from Figure 3). This implies that the loadings considered in this work are generally above the typical loadings observed in experimental systems, and well below the saturation or maximum attainable loadings. Also shown in Table 1 are the lengths of the control volumes used at each of the conditions stated before. Each simulation for the pore exit case is run at exactly the same fugacities as its corresponding simulation for the intracrystalline case. This means that the sorbate loadings in CV2 differ between the two types of simulations, due to the absence of attractive zeolite interactions in the pore exit simulations. However, the loading inside the pore at the zeolitebulk interface roughly corresponds to the equilibrium loading at the interfacial fugacity value, which is nearly equal to $f_{\mathrm{CV} 2}$. This means that the average loading inside the zeolite for the two corresponding sets of simulations differs only slightly, as shown in the next section.

The simulations were run for a time on the order of microseconds so as to obtain reliable steady-state fluxes. The lengths of the simulation runs varied with the simulation as shown in Table 1. The simulations at low temperatures were generally run for a longer period of time compared to those at moderate to high temperatures because fluxes are lower at low temperatures and thus harder to compute. The steady-state fluxes were recorded only after an initial simulation time of $t_{\text {init }}\left(t_{\text {init }}\right.$ $=0.25 \mu \mathrm{s}$ at $T=300,400$, and $500 \mathrm{~K} ; t_{\text {init }}=0.5 \mu \mathrm{s}$ at $T=100$ and $200 \mathrm{~K}$ ). The sorbate fluxes in the $z$ direction, $J_{z}$, were measured every time step via the flux plane method

$$
J_{z}=\frac{J^{\mathrm{LTR}}-J^{\mathrm{RTL}}}{t_{\mathrm{run}} A_{x y}}
$$

where $t_{\text {run }}$ is the simulation run time over which the fluxes are recorded and $A_{x y}$ is the cross-sectional area of the unit cell. $J^{\mathrm{LTR}}$ and $J^{\mathrm{RTL}}$ represent the net number of sorbate molecules that move left to right and right to left, respectively, through the flux plane situated at the middle of the right transport region. A second flux plane is placed at the middle of the left transport region, and the flux is measured in a similar way. The two resulting fluxes are then used to obtain an average flux.

\subsection{Simulation Results.}

Four sets of simulation runs were conducted at each of the prescribed conditions mentioned in the previous section. Each simulation started from a different initial configuration, and most of the results shown here are the averages of results from these four runs. One of the quantities calculated during each simulation run is the number density profile. This is calculated by dividing the axial length of the simulation box into a number of bins, where each bin is the width of a unit cell (i.e., the bin size is equal to $8.484 \AA$ in the $z$ direction). The average number of molecules in each bin, over the course of the simulation for $t>t_{\text {init }}$, is collected and then divided by the bin volume to give the density profile. The above bin size was chosen so as to remove the large fluctuations in the number density within each unit cell, which "camouflage" the overall nature of the density profile. These large density fluctuations are caused by the corrugations in the potential energy inside the pore. This results in sorbate molecules largely preferring to reside in the lowenergy regions within each unit cell, and being present only occasionally in the high-energy regions during hops between the potential minima.

Parts a and b of Figure 4 show the computed density profiles for the intracrystalline and pore exit simulation runs, respectively, at $\mathrm{L}, \mathrm{M}$, and $\mathrm{H}$ levels of loadings at $T=300 \mathrm{~K}$. We can observe that the loadings in the control volumes have been maintained fairly well. Also, the density profiles are fairly linear 

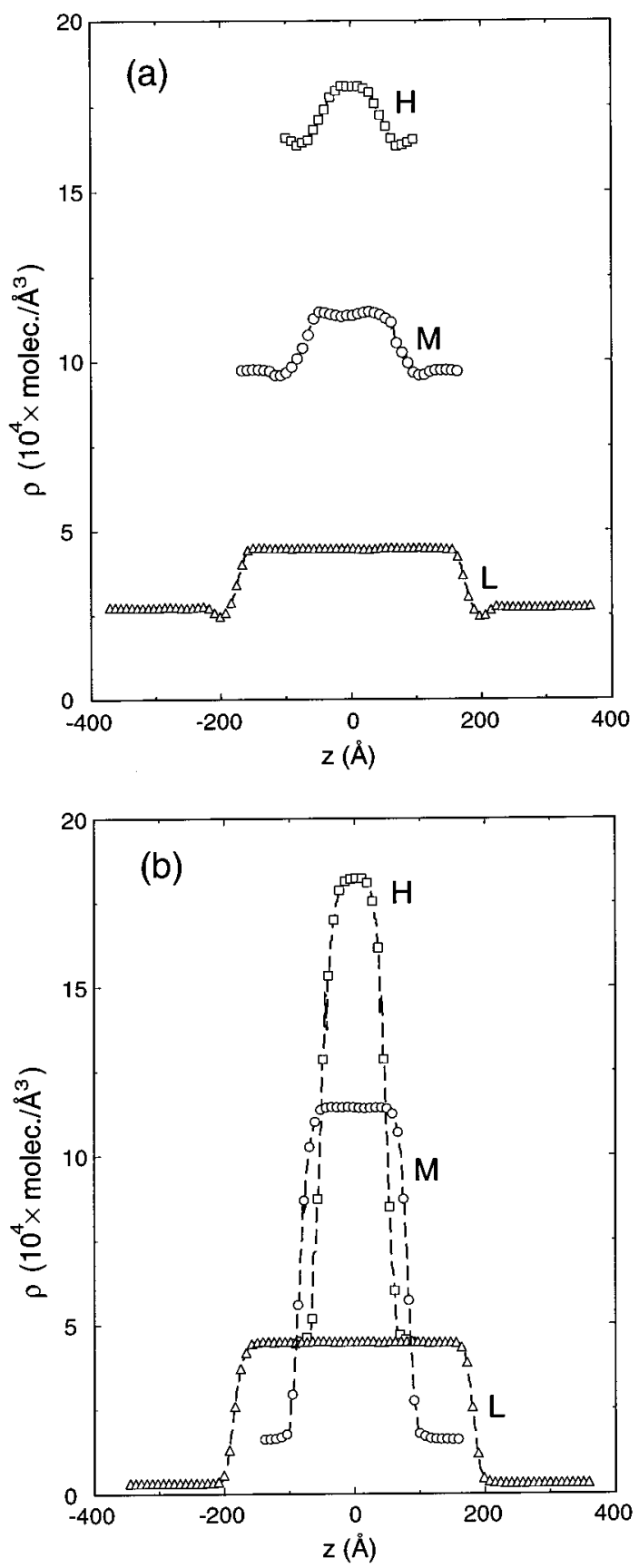

Figure 4. Density profile computed from the DCV-GCMD simulation runs for the (a) intracrystalline and (b) pore exit simulations at $T=$ $300 \mathrm{~K}$.

in the transport regions. The slight overshoot in the density profiles at the interface of the end control volumes and the transport regions in the intracrystalline simulations is probably due to the fact that the steady state has not been reached fully. Evidence for this is the fact that the low-temperature simulations (such as those performed at $T=100$ and $200 \mathrm{~K}$ ), which have very small transport diffusivities and thus reach steady state much slower, showed more pronounced overshoots in their density profiles. The density profiles obtained from simulations at the larger temperatures, $T=400$ and $500 \mathrm{~K}$, showed smaller and no overshoots in the density profile, respectively. The density profiles at the remaining temperatures have not been plotted as their basic nature remains the same as the ones shown here; i.e., they also show a linear behavior in the transport region.
Figure 5 shows the molecular fluxes calculated using eq 3, obtained for the intracrystalline and pore exit simulations conducted at different temperatures, and at the three different sorbate loadings, respectively. The fluxes have been multiplied by the cross-sectional area of the unit cell. The error bars in Figure 5 have been evaluated by taking the standard deviation of the four runs conducted at the same condition. The fluxes are also tabulated in Table 2. The fluxes in the positive and negative directions on the right and left halves, respectively, of the simulation cells for both the intracrystalline and pore exit simulations are identical within the statistical uncertainties of the results. Since the pore exit simulation cell is not perfectly symmetric, this indicates that the rate of escape of molecules is insensitive to the nature of the surface exposed to the bulk.

A direct comparison between the corresponding fluxes for the intracrystalline and the pore exit simulations cannot be made yet as the driving forces are different in the two cases. The true driving force for such diffusion problems is believed to be the chemical potential gradient, $\nabla_{z} \mu$. This is represented by the phenomenological equation

$$
J_{z}=-\mathrm{L} \nabla_{z} \mu
$$

where the transport coefficient $\mathrm{L}=\mathrm{L}(c, T)$ is a function of both sorbate loading $(c)$ and temperature $(T)$. We will use the gradient in the chemical potential as our driving force to evaluate the transport coefficient for all simulations. We evaluated the chemical potential profile in the transport region of the intracrystalline simulations using Widom's method ${ }^{20}$ and found it to be fairly linear, thus validating eq 4 for intracrystalline diffusion. The chemical potential profile in the pore exit region on the other hand was found to be very nonlinear and fluctuating, evidently due to the discontinuity of the potential at the pore mouth. By analogy with other interfacial masstransfer systems, we can define an effective or overall $\nabla_{z} \mu$ as

$$
\nabla_{z} \mu \approx \frac{R T \ln \left(f_{\mathrm{CV} 1} / f_{\mathrm{CV} 2}\right)}{l_{\mathrm{TR}}}
$$

where $R$ is the universal gas constant. The effective chemical potential gradients in the intracrystalline and pore exit regions computed using eq 5 are tabulated in Table 2.

Table 2 shows the calculated values of the effective transport coefficient, $L$, using eq 4 for the intracrystalline and pore exit regions at different temperatures and sorbate loadings. Also shown in Table 2 are the computed average sorbate loadings, $c_{\mathrm{av}}$, in the transport region. In the case of pore exit simulations, $c_{\mathrm{av}}$ is the average loading in the transport region that is inside the zeolite (and not in the bulk phase). It can be observed that the average loadings for the corresponding sets of simulations are only slightly different, so a fair comparison between the two sets of simulations can be made. Before the two sets of transport coefficients in Table 2 are compared, it is important to know whether the linearity between the flux and the chemical potential gradient is valid at some of the large chemical potential gradients used here. To check this, some of the intracrystalline simulations were run with longer transport region lengths, i.e., smaller chemical potential gradients. This way, only the chemical potential gradient was reduced without altering the average loading of the transport region (hence, $\mathrm{L}(c, T)$ should remain unaffected). Figure 6 shows one such set of fluxes obtained for $l_{\mathrm{TR}}=42.42,84.84,127.26$, and $169.68 \AA$ at $T=$ $400 \mathrm{~K}$ at the L level of sorbate loading. We can clearly observe from the figure that the flux $J_{z}$ increases linearly with $\nabla_{z} \mu$, hence validating eq 4 . 


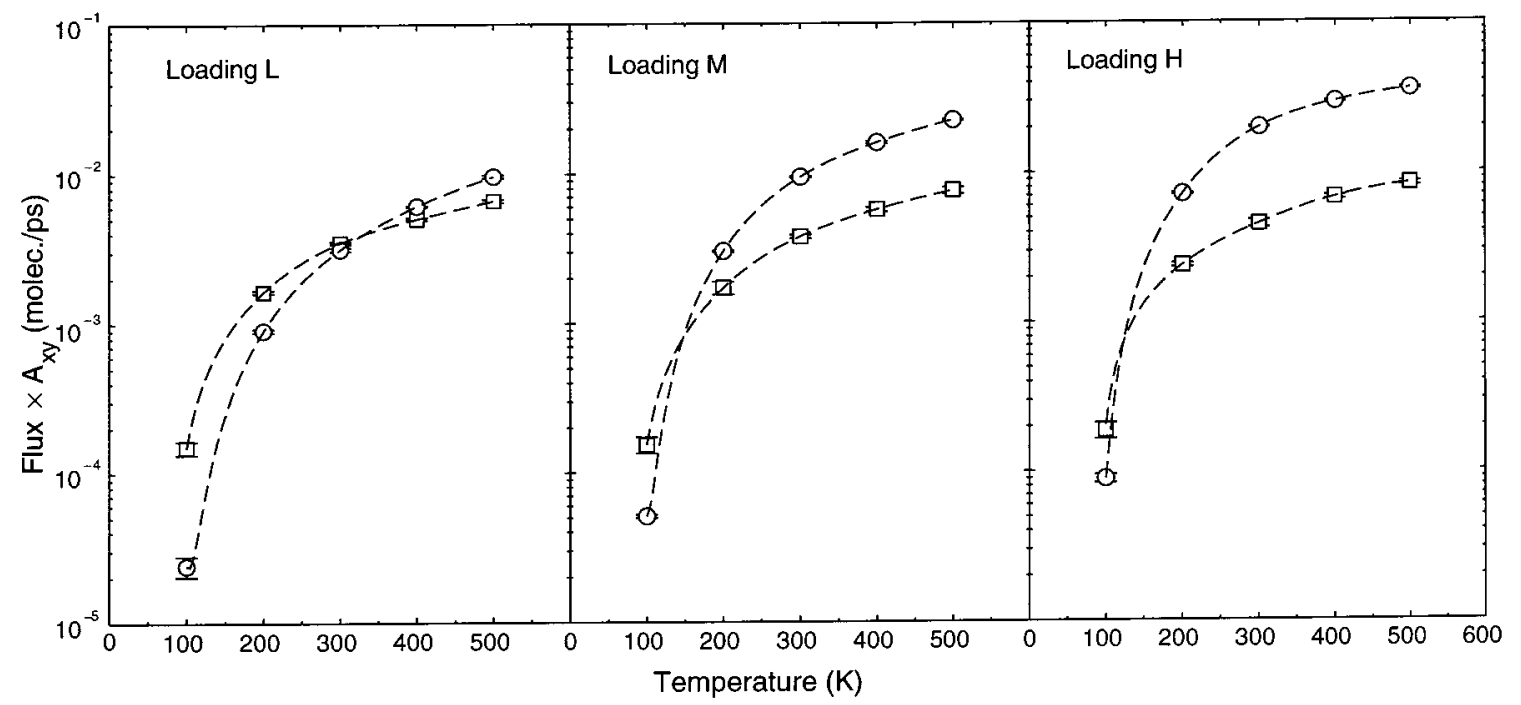

Figure 5. Fluxes obtained by DCV-GCMD simulations for L, M, and $\mathrm{H}$ levels of sorbate loadings at different temperatures for both the intracrystalline (shown by empty squares) and pore exit (shown by empty circles) cases. The dashed lines are intended to guide the eye.

TABLE 2: Measured Flux, $J_{z}$, Measured Average Loading Inside the Transport Region of the Pore, $c_{\mathrm{av}}$, Chemical Potential Gradient, $\Delta \mu_{z}$, and Calculated Transport Coefficient, L, for the DCV-GCMD Simulations for Both the Intracrystalline and the Pore Exit Cases ${ }^{a}$

\begin{tabular}{|c|c|c|c|c|c|}
\hline$T(\mathrm{~K})$ & $\begin{array}{c}\text { loading } \\
\text { level }\end{array}$ & $\begin{array}{c}J_{z} A_{x y} \\
\text { (molec./ps) }\end{array}$ & $\begin{array}{c}c_{\mathrm{av}} \\
\text { (molec./u. c.) }\end{array}$ & $\begin{array}{c}-\nabla \mu_{z} \\
(\mathrm{~J} / \mathrm{mol} / \mathrm{m})^{b}\end{array}$ & $\underset{\mathrm{m} / \mathrm{s} / \mathrm{J})}{\mathrm{L}_{\left(\mathrm{mol}^{2} /\right.}}$ \\
\hline \multicolumn{6}{|c|}{ Intracrystalline Simulations } \\
\hline \multirow[t]{3}{*}{100} & $\mathrm{~L}$ & $1.49(15) \times 10^{-4}$ & 0.43 & 6.02 & $2.52 \times 10^{-9}$ \\
\hline & M & $1.54(19) \times 10^{-4}$ & 1.33 & 1.53 & $1.02 \times 10^{-8}$ \\
\hline & $\mathrm{H}$ & $1.89(25) \times 10^{-4}$ & 2.32 & 1.19 & $1.61 \times 10^{-8}$ \\
\hline \multirow[t]{3}{*}{200} & $\mathrm{~L}$ & $1.65(04) \times 10^{-3}$ & 0.43 & 20.51 & $8.19 \times 10^{-9}$ \\
\hline & $\mathrm{M}$ & $1.73(17) \times 10^{-3}$ & 1.42 & 8.07 & $2.18 \times 10^{-8}$ \\
\hline & $\mathrm{H}$ & $2.38(07) \times 10^{-3}$ & 2.37 & 7.36 & $3.29 \times 10^{-8}$ \\
\hline \multirow[t]{3}{*}{300} & $\mathrm{~L}$ & $3.51(07) \times 10^{-3}$ & 0.48 & 33.47 & $1.07 \times 10^{-8}$ \\
\hline & M & $3.79(11) \times 10^{-3}$ & 1.42 & 15.41 & $2.50 \times 10^{-8}$ \\
\hline & $\mathrm{H}$ & $4.48(26) \times 10^{-3}$ & 2.47 & 13.46 & $3.39 \times 10^{-8}$ \\
\hline \multirow[t]{3}{*}{400} & $\mathrm{~L}$ & $5.04(09) \times 10^{-3}$ & 0.49 & 46.94 & $1.09 \times 10^{-8}$ \\
\hline & M & $5.72(21) \times 10^{-3}$ & 1.46 & 22.68 & $2.57 \times 10^{-8}$ \\
\hline & $\mathrm{H}$ & $6.70(20) \times 10^{-3}$ & 2.48 & 20.16 & $3.38 \times 10^{-8}$ \\
\hline \multirow[t]{3}{*}{500} & $\mathrm{~L}$ & $6.63(16) \times 10^{-3}$ & 0.51 & 59.34 & $1.14 \times 10^{-8}$ \\
\hline & M & $7.63(39) \times 10^{-3}$ & 1.48 & 29.43 & $2.64 \times 10^{-8}$ \\
\hline & $\mathrm{H}$ & $8.28(26) \times 10^{-3}$ & 2.52 & 26.05 & $3.23 \times 10^{-8}$ \\
\hline \multicolumn{6}{|c|}{ Pore Exit Simulations } \\
\hline \multirow[t]{3}{*}{100} & $\mathrm{~L}$ & $2.41(38) \times 10^{-5}$ & 0.39 & 7.52 & $3.26 \times 10^{-10}$ \\
\hline & M & $5.12(13) \times 10^{-5}$ & 1.28 & 1.92 & $2.71 \times 10^{-9}$ \\
\hline & $\mathrm{H}$ & $8.88(56) \times 10^{-5}$ & 2.25 & 1.49 & $6.01 \times 10^{-9}$ \\
\hline \multirow[t]{3}{*}{200} & $\mathrm{~L}$ & $9.05(25) \times 10^{-4}$ & 0.44 & 25.64 & $3.59 \times 10^{-9}$ \\
\hline & M & $3.05(04) \times 10^{-3}$ & 1.39 & 10.09 & $3.08 \times 10^{-8}$ \\
\hline & $\mathrm{H}$ & $7.13(08) \times 10^{-3}$ & 2.27 & 9.20 & $7.89 \times 10^{-8}$ \\
\hline \multirow[t]{3}{*}{300} & $\mathrm{~L}$ & $3.17(08) \times 10^{-3}$ & 0.48 & 41.84 & $7.71 \times 10^{-9}$ \\
\hline & $\mathrm{M}$ & $9.50(13) \times 10^{-3}$ & 1.39 & 19.26 & $5.02 \times 10^{-8}$ \\
\hline & $\mathrm{H}$ & $1.47(02) \times 10^{-2}$ & 2.28 & 16.82 & $8.89 \times 10^{-8}$ \\
\hline \multirow[t]{3}{*}{400} & $\mathrm{~L}$ & $6.14(06) \times 10^{-3}$ & 0.48 & 58.68 & $1.06 \times 10^{-8}$ \\
\hline & M & $1.60(03) \times 10^{-2}$ & 1.44 & 28.35 & $5.74 \times 10^{-8}$ \\
\hline & $\mathrm{H}$ & $2.92(04) \times 10^{-2}$ & 2.39 & 25.20 & $1.18 \times 10^{-7}$ \\
\hline \multirow[t]{3}{*}{500} & $\mathrm{~L}$ & $9.64(24) \times 10^{-3}$ & 0.49 & 74.17 & $1.32 \times 10^{-8}$ \\
\hline & M & $2.25(02) \times 10^{-2}$ & 1.47 & 36.79 & $6.22 \times 10^{-8}$ \\
\hline & $\mathrm{H}$ & $3.55(06) \times 10^{-2}$ & 2.48 & 32.57 & $1.11 \times 10^{-7}$ \\
\hline
\end{tabular}

${ }^{a}$ The values within parentheses represent the statistical uncertainties in the last reported digits. ${ }^{b}$ Values to $10^{10}$.

The contribution of the surface barrier to the total resistance obviously depends on the crystal dimension or intracrystalline pore length. Hence, any assessment of the surface barrier resistance must include an estimate of the critical crystal dimension beyond which the barrier resistance becomes insignificant. One way to quantify this relative importance of the surface barrier is to evaluate the ratio of the intracrystalline length of the pore $\left(z_{\mathrm{I}}\right)$ to the length of the pore exit region $\left(z_{\mathrm{E}}\right)$

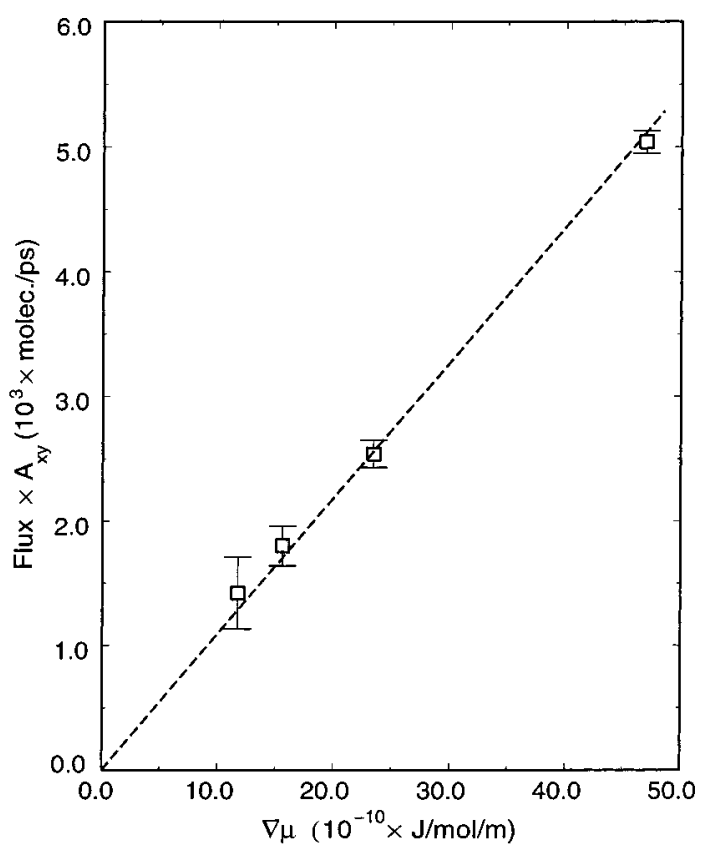

Figure 6. Intracrystalline fluxes at $\mathrm{L}$ sorbate loading obtained at $T=$ $400 \mathrm{~K}$ at $l_{\mathrm{TR}}=42.42,84.84,127.26$, and $169.68 \AA$, plotted against the corresponding chemical potential gradients. The dashed line is a linear fit to the above data forced to pass through the origin.

required so that the intracrystalline resistance becomes equal to the surface barrier resistance. The governing equation for the flux in the intracrystalline region of the pore is given by

$$
J_{z}=-\mathrm{L}_{\mathrm{I}} \frac{\left(\Delta \mu_{z}\right)_{\mathrm{I}}}{z_{\mathrm{I}}}
$$

and at the pore exit by

$$
J_{z}=-\mathrm{L}_{\mathrm{E}} \frac{\left(\Delta \mu_{z}\right)_{\mathrm{E}}}{z_{\mathrm{E}}}
$$

where $L_{I}$ and $L_{E}$ are the effective transport coefficients in the intracrystalline region and the pore exit, respectively (as calculated separately from the two sets of simulations in this work). Since the transport resistances related to the intracrystalline region and the pore exit are in series, the total resistance 


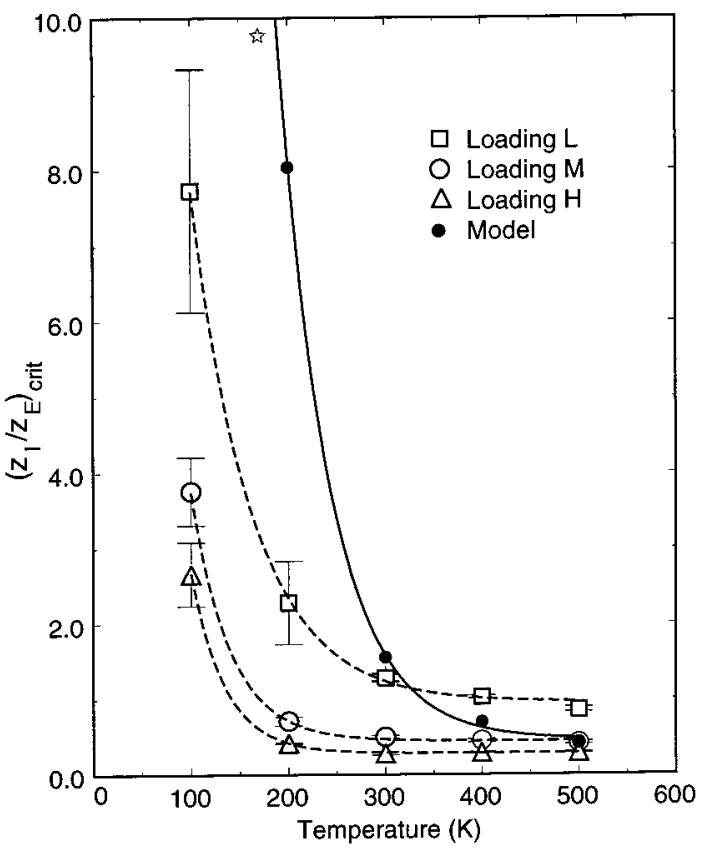

Figure 7. Relative importance of the surface barrier resistance to the intracrystalline resistance, $\left(z_{\mathrm{I}} / z_{\mathrm{E}}\right)_{\text {crit }}$, plotted against the temperature at different sorbate loadings. Also shown are the $\left(z_{\mathrm{I}} / z_{\mathrm{E}}\right)_{\text {crit }}$ values predicted by the activated transport model. The star symbol implies that the value of $\left(z_{1} / z_{\mathrm{E}}\right)_{\text {crit }}$ predicted by the model at $T=100 \mathrm{~K}$ is too large to fit in the present plot. The lines are meant to guide the eye.

in a pore containing both the intracrystalline and pore exit regions is given by the sum of the two resistances, whereby we obtain

$$
J_{z}=-\mathrm{L}_{\mathrm{I}} \frac{\left(\Delta \mu_{z}\right)_{\mathrm{T}}}{z_{\mathrm{I}}} /\left(1+\frac{z_{\mathrm{E}} \mathrm{L}_{\mathrm{I}}}{z_{\mathrm{I}} \mathrm{L}_{\mathrm{E}}}\right)
$$

where $\left(\Delta \mu_{z}\right)_{\mathrm{T}}=\left(\Delta \mu_{z}\right)_{\mathrm{E}}+\left(\Delta \mu_{z}\right)_{\mathrm{I}}$ is the total chemical potential difference across the intracrystalline and pore exit regions. We consider that the exit effects become dominating when the ratio of the intracrystalline length to the length of the exit region, $z_{\mathrm{I}} / z_{\mathrm{E}}$, becomes less than the ratio of the two transport coefficients, $L_{I} / L_{E}$. Therefore, exit effects become important when $z_{\mathrm{I}} / z_{\mathrm{E}}<\left(z_{\mathrm{I}} / z_{\mathrm{E}}\right)_{\text {crit }}$, where the critical ratio $\left(z_{\mathrm{I}} / z_{\mathrm{E}}\right)_{\text {crit }}$ is equal to $\mathrm{L}_{\mathrm{I}} / \mathrm{L}_{\mathrm{E}}$.

Figure 7 is a plot of $\left(z_{\mathrm{I}} / z_{\mathrm{E}}\right)_{\text {crit }}$ versus temperature at the three sorbate loadings investigated in this paper. It is observed that the ratio $\left(z_{\mathrm{I}} / z_{\mathrm{E}}\right)_{\text {crit }}$ decays rapidly as the temperature increases. This indicates that the presence of a pore exit hinders the rate of diffusion at low temperatures, and that exit effects become less important as temperature increases. This can be understood by noting that the energy barrier with respect to the thermal energy increases drastically as the temperature decreases (as shown using the potential of mean force in the next section), thus retarding the molecules at the pore mouth. This effect of the pore exit on the energy barrier is, interestingly, much more pronounced when the sorbate loading in the zeolite is low than at high sorbate loadings. This could be due to the fact that, at high sorbate loadings, the $\mathrm{CH}_{4}$ molecules drift down the chemical potential gradient in local clusters. ${ }^{21} \mathrm{~A}$ molecule inside the pore, but near the exit, is aided by its neighbors behind it, pushing it to cross over the energy barrier, thus effectively reducing the activation barrier for transport. At low loadings, however, the sorbate-sorbate interactions cease to exist as the molecules are too far apart to affect the motion of one another. Therefore, a molecule will escape from the pore only when it has gathered enough momentum in the axial direction from its radial degrees of freedom to jump over the energy barrier at the pore exit. ${ }^{22}$ A surprising observation is that the transport coefficients at $T>100 \mathrm{~K}$ for sorbate loadings $\mathrm{M}$ and $\mathrm{H}$ and $T$ $>400 \mathrm{~K}$ for sorbate loading $\mathrm{L}$ are in fact larger in the pore exit case than in the intracrystalline case. The origin of this unusual behavior, first predicted to exist by Kärger, ${ }^{10}$ will be explained in the next section in terms of a simple activated transport model. From the above analysis, the computed $\left(z_{\mathrm{I}} / z_{\mathrm{E}}\right)_{\text {crit }}$ does not exceed 10 even at the lowest temperature and loading. This implies that, for a crystal dimension exceeding $10 z_{\mathrm{E}} \approx 340 \AA$, surface barrier resistance would no longer make a significant contribution to the total resistance of an $\mathrm{AlPO}_{4}-5$ pore.

The DCV-GCMD simulations thus enable one to directly compute the relative importance of the exit barrier on the transport of sorbates across zeolite pores at different temperatures and loadings. However, the simulations are computationally demanding (many of the simulations conducted here took over 4 days of CPU time on SunSPARC ULTRA 30 computers), and are impractical for systems having much smaller transport rates than methane. To examine these systems, we must use coarser-grained models. In the next section we develop a simple model based on activated transport to study the importance of the pore exit for a range of sorbate molecules in $\mathrm{AlPO}_{4}-5$.

\section{Activated Transport Theory}

\subsection{Model Details.}

In our model, we assume thermodynamic equilibrium in the transverse direction of the long pores. Longitudinal variations of the potential and pore spacing then produce a pseudo-onedimensional potential landscape over which the sorbate is driven by some kind of thermal noise. Key to this model is the large entropy gradient at the exit, which may compensate the favorable adsorptive potential of the crystal.

We now assume activated transport of sorbate molecules along the longitudinal periodic energy barriers in the intracrystalline region of the pore and surface barrier at the pore exit. Therefore, the diffusivity or mobility in the intracrystalline region, $L_{I}$, scales in an Arrhenius fashion with the temperature:

$$
\mathrm{L}_{\mathrm{I}} \approx \exp \left(-\Delta U_{\mathrm{I}} / k_{\mathrm{B}} T\right)
$$

where $\Delta U_{\mathrm{I}}$ is the intracrystalline activation barrier. For the pore exit region, a similar scaling law for the mobility, $L_{E}$, also applies:

$$
\mathrm{L}_{\mathrm{E}} \approx \exp \left(-\Delta U_{\mathrm{E}} / k_{\mathrm{B}} T\right)
$$

where $\Delta U_{\mathrm{E}}$ is the pore exit or surface barrier. Although thermal noise is not introduced in our MD simulations and our static lattice does not yield thermal vibrations, the transport of a single sorbate is still diffusive with random walk characteristics. There are two possible origins to this stochastic motion. One is dynamic interaction (collision) among sorbate molecules, which can be significant even at low loadings. The dynamics of a neighboring sorbate two or three unit cells away can produce a dynamic potential. The interaction can also produce deterministic clustering. Another possibility is that the motion of the sorbate in the pore cross-section transverse to the longitudinal diffusive $z$ direction can produce random motion in the $z$ direction. This coupling between the transverse degrees of freedom with the dynamics in the $z$ direction has been explored by Kopelevich and Chang. ${ }^{22}$ We model both dynamics as thermal noise here with the activated transport rate. However, the frequency factor is unknown, and hence only relative fluxes can be estimated. 

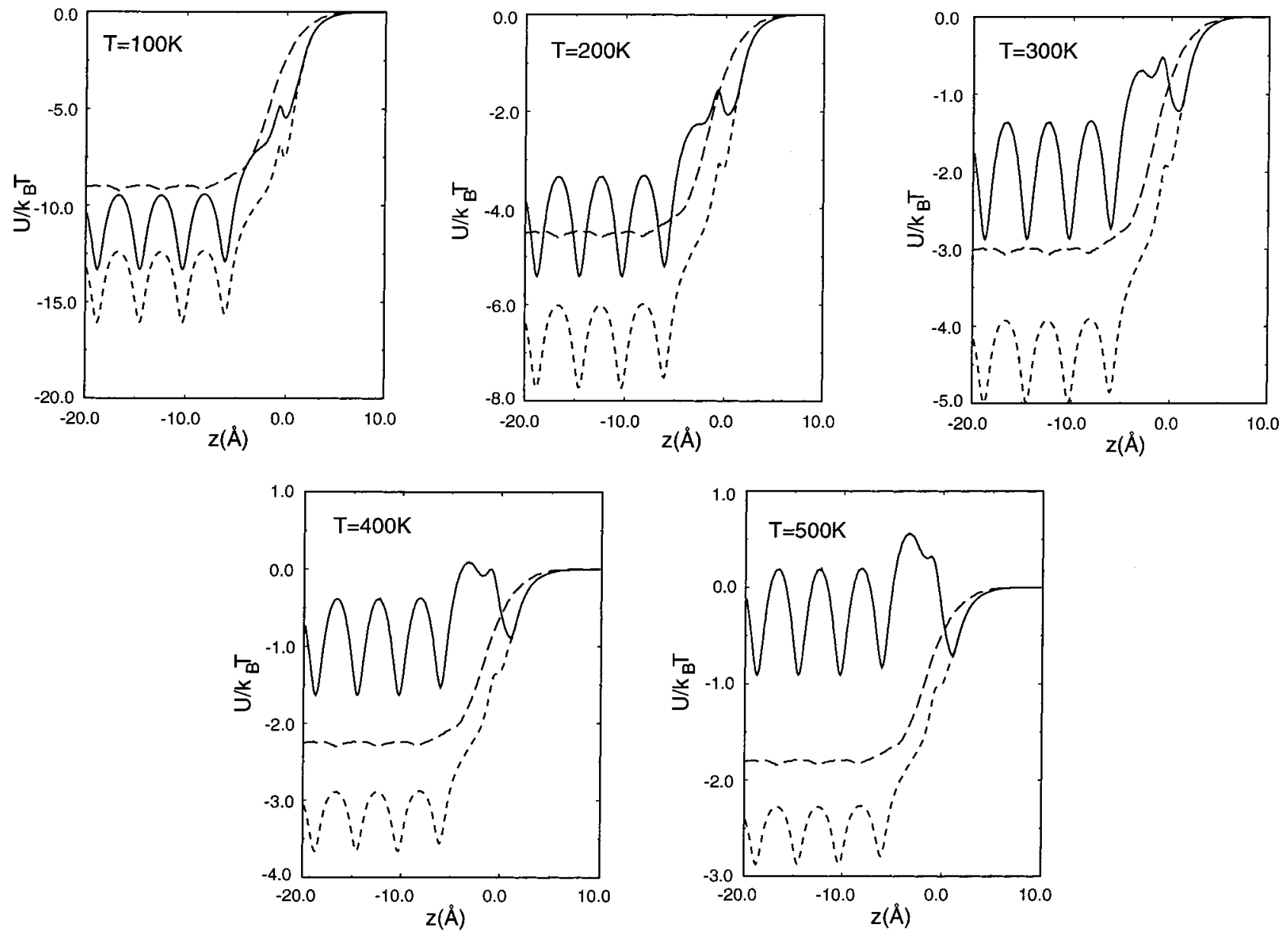

Figure 8. Potentials of mean force for methane, shown as solid lines, plotted at $T=100,200,300,400$, and $500 \mathrm{~K}$ as a function of the axial coordinate, $z$. Also shown are the average Boltzmann-weighted potential profiles, shown by dotted lines, and the potentials at the central axis of the pore, shown by dashed lines.

As before, the exit resistance would dominate over the intracrystalline resistance when the ratio of the intracrystalline length $\left(z_{\mathrm{I}}\right)$ and the exit region length $\left(z_{\mathrm{E}}\right)$ is less than $\mathrm{L}_{\mathrm{I}} / \mathrm{L}_{\mathrm{E}}$. This critical ratio of the two lengths, $\left(z_{\mathrm{I}} / z_{\mathrm{E}}\right)_{\text {crit }}$, is then given by

$$
\left(z_{\mathrm{I}} / z_{\mathrm{E}}\right)_{\text {crit }}=\mathrm{L}_{\mathrm{I}} / \mathrm{L}_{\mathrm{E}} \approx \exp \left(-\Delta E / k_{\mathrm{B}} T\right)
$$

where $\Delta E=\Delta U_{\mathrm{I}}-\Delta U_{\mathrm{E}}$ is the difference in the activation barrier heights in the intracrystalline and pore exit regions. The activation barriers used in the model should properly account for the potential energy as well as entropy. To this end, we use barriers extracted from the potential of mean force (PMF).

The $\mathrm{PMF}^{23,24}$ for an $N$-particle system keeping $n$ of these particles fixed at positions $\mathbf{r}_{1}, \ldots, \mathbf{r}_{n}$ is defined in terms of the $n$-particle distribution function $g^{(n)}\left(\mathbf{r}_{1}, \ldots, \mathbf{r}_{n}\right)$ as

$$
W^{(n)}\left(\mathbf{r}_{1}, \ldots, \mathbf{r}_{n}\right)=-k_{\mathrm{B}} T \ln g^{(n)}\left(\mathbf{r}_{1}, \ldots, \mathbf{r}_{n}\right)
$$

where

$g^{(n)}\left(\mathbf{r}_{1}, \ldots, \mathbf{r}_{n}\right)=\frac{V^{n} N !}{N^{n}(N-n) !} \frac{\int \ldots \int \mathrm{e}^{-\beta v\left(\mathbf{r}_{1}, \ldots, \mathbf{r}_{N}\right)} \mathrm{d} \mathbf{r}_{n+1} \ldots \mathrm{d} \mathbf{r}_{N}}{\int \ldots \int \mathrm{e}^{-\beta v\left(\mathbf{r}_{1}, \ldots, \mathbf{r}_{N}\right)} \mathrm{d} \mathbf{r}_{1} \ldots \mathrm{d} \mathbf{r}_{N}}$

Here $\beta=1 / k_{\mathrm{B}} T$, with $k_{\mathrm{B}}$ being the Boltzmann constant and $T$ the temperature, and $v\left(\mathbf{r}_{1}, \ldots, \mathbf{r}_{N}\right)$ is the potential energy. The integral in the denominator is the entire configurational integral, while the integral in the numerator is over all conformations keeping $\mathbf{r}_{1}, \ldots, \mathbf{r}_{n}$ fixed.

Now consider a single sorbate molecule inside an $\mathrm{AlPO}_{4}-5$ pore. Keeping the $z$ coordinate of the sorbate fixed and varying the position of the molecule in the $x-y$ plane of the unit cell, the PMF can be calculated as a function of location along the pore axis $(z)$ as

$W(z)=-k_{\mathrm{B}} T \ln g(z)=-k_{\mathrm{B}} T \ln \left(\frac{L \iint \mathrm{e}^{-\beta v(x, y, z)} \mathrm{d} x \mathrm{~d} y}{\iiint \mathrm{e}^{-\beta v(x, y, z)} \mathrm{d} x \mathrm{~d} y \mathrm{~d} z}\right)$

where $L$ is the length of the pore being investigated. Here the integrations in the numerator are over the entire unit cell cross sections in the $x-y$ plane at axial position, $z$, whereas the integrations in the denominator span the total zeolite volume considered in the analysis. The resulting PMF is the free energy profile of a molecule diffusing along the $z$ axis, appropriately averaged over the coordinates orthogonal to the pore axis. To compute the PMF in practice, a Monte Carlo integration technique is performed, with integrations carried out at small intervals of $0.1 \AA$ along the $z$ axis.

Figure 8 shows the computed PMF profiles for methane at five different temperatures for a sorbate near the pore exit. The zeolite-bulk interface is at $z=0$, while the regions inside the pore are at $z<0$ and the regions in the bulk are at $z>0$. When the sorbate is well inside the pore region (i.e., it does 
not "feel" the pore exit), the PMF is a series of periodic "bumps", which correspond to the natural corrugation of the zeolite potential field induced by the atomistic roughness of the pore wall. The amplitude of these PMF corrugations yields the activation energy barrier, $\Delta U_{\mathrm{I}}$, in the intracrystalline region of the pore. As the molecule approaches the pore exit, the attractive interactions between the sorbate and the zeolite lattice begin to decrease, resulting in an increase in the PMF. This is the origin of the pore exit barrier. Comparing the PMF profile at progressively higher temperatures in Figure 8, it is clearly observed that the barrier height relative to $k_{\mathrm{B}} T$ decreases, consistent with the simulation results. The results obtained using PMF predict that the exit barrier should be most severe at low temperatures and could potentially be the rate-limiting step for intercrystalline transport. The height of the pore exit barrier relative to the mean $\mathrm{PMF}$ in the intracrystalline region gives the activation energy barrier for the pore exit, as denoted by $\Delta U_{\mathrm{E}}$. Before computing $\left(z_{\mathrm{I}} / z_{\mathrm{E}}\right)_{\text {crit }}$ using the two activation barriers, we would like to compare the PMF with two other forms of potential in the pore.

Also shown in Figure 8 as dotted lines are the variations in the average potential energy along the pore axis, $\langle v(z)\rangle_{\mathrm{NVT}}$, calculated by taking a Boltzmann weighted average of the potential:

$$
\langle v(z)\rangle_{\mathrm{NVT}}=\frac{\iint v(x, y, z) \mathrm{e}^{-\beta v(x, y, z)} \mathrm{d} x \mathrm{~d} y}{\iint \mathrm{e}^{-\beta v(x, y, z)} \mathrm{d} x \mathrm{~d} y}
$$

Note that $\langle v(z)\rangle_{\mathrm{NVT}}$ shows a corrugation similar to that of $W(z)$, but the exit barrier for the average potential energy is higher than that for the PMF. This can be understood by virtue of the fact that $W(z)$ is a free energy whereas $\langle v(z)\rangle_{\mathrm{NVT}}$ only accounts for potential energy variations. The sorbate experiences an increase in the potential energy as it leaves the strong energetic field induced by the zeolite, but it also experiences an increase in configurational (entropic) freedom. This increased entropic freedom serves to lower the free energy barrier and reduces the pore exit contribution more than one expects from purely energetic arguments. Finally, the potential energy profile of a sorbate as it is dragged along the axis of the pore structure is shown in Figure 8 as a dashed line. This potential shows no corrugation within the internal pore region, reflecting the fact that this corrugation arises from the intimate contact with the atoms comprising the pore wall. Note that the potential energy at the center of the pore is actually higher than $\langle v(z)\rangle_{\mathrm{NVT}}$, which reflects the fact that methane prefers to "hug" the pore walls of $\mathrm{AlPO}_{4}-5$ to maximize favorable van der Waals interactions with the lattice. The potential energy at the pore center gradually increases as the pore exit is approached, which indicates the diminishing impact of the lattice near the pore exit. The differences in the activation barriers in the intracrystalline and pore exit regions of the pore $(\Delta E)$ calculated using the three different techniques at the five different temperatures are listed in Table 3.

\subsection{Model Results.}

Having obtained $\Delta U_{\mathrm{I}}$ and $\Delta U_{\mathrm{E}}$, we now evaluate $\left(z_{\mathrm{I}} / z_{\mathrm{E}}\right)_{\text {crit }}$ using eq 11 at temperatures ranging from $T=100 \mathrm{~K}$ to $T=$ $500 \mathrm{~K}$ for the single methane molecule in $\mathrm{AlPO}_{4}-5$. The values of $\left(z_{\mathrm{I}} / z_{\mathrm{E}}\right)_{\text {crit }}$ for methane computed using the model have been plotted in Figure 7, alongside the results from simulations. The values are also listed in Table 4. It is observed that the general trend in $\left(z_{\mathrm{I}} / z_{\mathrm{E}}\right)_{\text {crit }}$ obtained from our model is similar to that obtained via the simulations; i.e., here also $\left(z_{\mathrm{I}} / z_{\mathrm{E}}\right)_{\text {crit }}$ decays with temperature, showing a diminishing importance of exit effects
TABLE 3: Energy Barrier Difference, $\Delta E$, for Methane Evaluated at Different Temperatures from the PMF $\left(\Delta E^{\mathrm{P}}\right)$, the Boltzmann-Averaged Potential $\left(\Delta E^{\mathrm{M}}\right)$, and the Potential at the Center of the Pore $\left(\Delta E^{\mathrm{C}}\right)^{a}$

\begin{tabular}{cccc}
\hline$T(\mathrm{~K})$ & $\Delta E^{\mathrm{P}} / k_{\mathrm{B}} T$ & $\Delta E^{\mathrm{M}} / k_{\mathrm{B}} T$ & $\Delta E^{\mathrm{C}} / k_{\mathrm{B}} T$ \\
\hline 100 & 10.88 & 13.71 & 9.05 \\
200 & 4.12 & 6.62 & 4.53 \\
300 & 1.94 & 4.31 & 3.02 \\
400 & 0.87 & 3.17 & 2.26 \\
500 & 0.24 & 2.49 & 1.81
\end{tabular}

${ }^{\text {a }}$ Each $\Delta E$ has been normalized by $k_{\mathrm{B}} T$.

with temperature. The values of $\left(z_{\mathrm{I}} / z_{\mathrm{E}}\right)_{\text {crit }}$ obtained from the theoretical model are in good agreement with those obtained from the simulation at loading $\mathrm{L}$ for most of the temperatures. Only at $T=100 \mathrm{~K}$ is the discrepancy between the two results very large. At this temperature, the value of $\left(z_{\mathrm{I}} / z_{\mathrm{E}}\right)_{\text {crit }}$ obtained from the simulations is about 2 orders of magnitude smaller than that obtained from our model. It is not clear as to the exact source of the discrepancy, but there are three possible factors that may be responsible.

First, the projection of sorbate trajectories onto a onedimensional path along the pore axis may be invalid near the pore exit, as the molecules are no longer confined to move along the $z$ axis. Second, the local equilibrium assumption made in the activated transport model could also be flawed. Third, and most importantly, the present model neglects interaction among sorbate molecules. In the DCV-GCMD simulations, however, the sorbate molecules interact with each other and tend to form clusters, especially at low temperatures. It has been recently observed $^{21}$ that activation barriers for diffusion are significantly reduced due to cluster formation. The impact of the clustering is greatest at the pore exit where the energy barriers are quite high. This would lead to a significant increase in fluxes at the exit and could possibly explain why the proposed model overpredicts the exit effects at low temperatures. Clearly these issues require further examination.

We now apply this model to study the importance of exit effects for the transport of other molecules in $\mathrm{AlPO}_{4}-5$ pores. This way we can examine the effect of the molecular size parameter, $\sigma$, and interaction parameter, $\epsilon$, on the above phenomena. The molecules chosen for our analysis, with their size and interaction parameters, taken from ref 25 , are listed in Table 4. Interactions of sorbates with the $\mathrm{AlPO}_{4}-5$ lattice were modeled using Lorentz-Berthelot combining rules with the oxygen LJ parameters given by $\sigma_{\mathrm{O}}=2.698 \AA$ and $\epsilon_{\mathrm{O}} / k_{\mathrm{B}}=120.1$ $\mathrm{K}$. The PMF for these molecules is calculated at the five temperatures as in the case of methane. Figure 9 shows the PMF of the seven molecules at one particular temperature $(T=300$ $\mathrm{K})$. To evaluate $\left(z_{\mathrm{I}} / z_{\mathrm{E}}\right)_{\text {crit }}$, the activation energies in the intracrystalline and pore exit regions are obtained from these PMFs. Table 4 shows the computed values of $\left(z_{\mathrm{I}} / z_{\mathrm{E}}\right)_{\text {crit }}$ for the various sorbates considered. It can be observed that, for all the molecules, the exit effects become less important as the temperature is raised, as expected. Another important observation is the fact that, as the molecular size becomes large, the exit effects become more important. These trends can be observed more clearly by plotting $\left(z_{\mathrm{I}} / z_{\mathrm{E}}\right)_{\text {crit }}$ at different temperatures for the various molecules considered in the same figure (Figure 10). It is observed that the relative importance of the barrier at the exit for the various molecules decreases in the order $\mathrm{SnBr}_{4}>\mathrm{CCl}_{4}>\mathrm{CF}_{4}>\mathrm{Xe}>\mathrm{CH}_{4}>\mathrm{Ar}>\mathrm{Ne}$. We notice that this is identical to the order in which these molecules would be arranged if they were to be placed in decreasing order of molecular size. Figure 9 also shows a similar trend as the 
TABLE 4: Lennard-Jones Parameters for Sorbate Molecules and the Relative Importance of Exit Effects for Different Molecules in an $\mathrm{AlPO}_{4}-5$ Pore Using the Activated Transport Model

\begin{tabular}{|c|c|c|c|c|c|c|c|}
\hline & \multicolumn{2}{|c|}{ LJ parameters } & \multicolumn{5}{|c|}{$\left(z_{\mathrm{I}} / z_{\mathrm{E}}\right)_{\text {crit }}$ computed from the activated transport model } \\
\hline & $\overline{\sigma(\AA)}$ & $\epsilon / k_{\mathrm{B}}(\mathrm{K})$ & $T=100 \mathrm{~K}$ & $T=200 \mathrm{~K}$ & $T=300 \mathrm{~K}$ & $T=400 \mathrm{~K}$ & $T=500 \mathrm{~K}$ \\
\hline $\mathrm{SnBr}_{4}$ & 6.666 & 465.0 & $6.97 \times 10^{34}$ & $2.16 \times 10^{16}$ & $1.77 \times 10^{10}$ & $1.97 \times 10^{7}$ & $2.38 \times 10^{5}$ \\
\hline $\mathrm{CCl}_{4}$ & 5.881 & 327.0 & $1.65 \times 10^{23}$ & $2.40 \times 10^{10}$ & $1.66 \times 10^{6}$ & $1.27 \times 10^{4}$ & $8.09 \times 10^{2}$ \\
\hline $\mathrm{CF}_{4}$ & 4.70 & 152.5 & $1.33 \times 10^{7}$ & $6.10 \times 10^{2}$ & $2.51 \times 10^{1}$ & $4.77 \times 10^{0}$ & $1.76 \times 10^{0}$ \\
\hline $\mathrm{Xe}$ & 4.055 & 229.0 & $3.70 \times 10^{5}$ & $1.36 \times 10^{2}$ & $9.22 \times 10^{0}$ & $2.50 \times 10^{0}$ & $1.14 \times 10^{0}$ \\
\hline $\mathrm{CH}_{4}$ & 3.73 & 147.95 & $1.12 \times 10^{3}$ & $8.04 \times 10^{0}$ & $1.55 \times 10^{0}$ & $7.00 \times 10^{-1}$ & $4.18 \times 10^{-1}$ \\
\hline $\mathrm{Ar}$ & 3.418 & 124.0 & $9.76 \times 10^{1}$ & $2.30 \times 10^{0}$ & $7.57 \times 10^{-1}$ & $3.92 \times 10^{-1}$ & $2.82 \times 10^{-1}$ \\
\hline $\mathrm{Ne}$ & 2.789 & 35.7 & $4.35 \times 10^{-1}$ & $2.33 \times 10^{-1}$ & $1.69 \times 10^{-1}$ & $1.47 \times 10^{-1}$ & $1.33 \times 10^{-1}$ \\
\hline
\end{tabular}

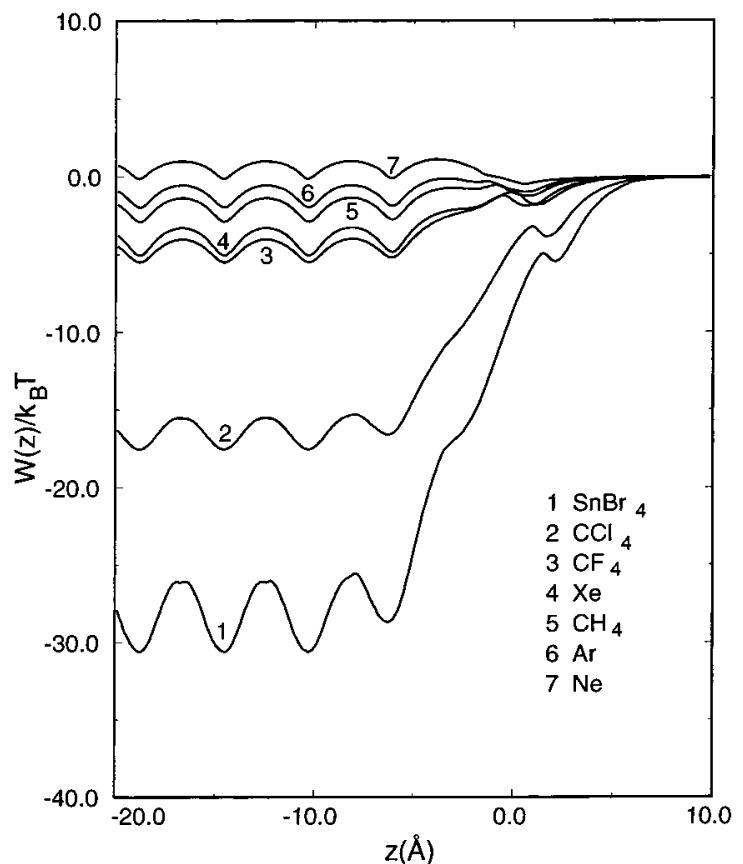

Figure 9. Potential of mean force for the various molecules considered in this study at $T=300 \mathrm{~K}$.

exit barrier heights for the various molecules decrease in the same order. This shows that molecular size (or, more properly, the ratio of molecular size to pore diameter) is clearly the most relevant parameter governing the importance of exit effects in pores. Of course, a large $\epsilon$ would also enhance the relative importance of the pore exit, as this would serve to further increase the attractive interactions between the sorbate and the lattice.

To gauge the importance of exit effects on real systems, we assume that the length of a typical zeolite crystal is on the order of a micrometer, and that the length of a pore exit is about 34 $\AA$ (as used in our analysis before). This means that, for all systems with $\left(z_{\mathrm{I}} / z_{\mathrm{E}}\right)_{\text {crit }}$ greater than about 300 , exit effects may be important. We can therefore conclude, from Table 4 and Figure 10, that, at room temperature, exit effects are important for the transport of $\mathrm{SnBr}_{4}$ and $\mathrm{CCl}_{4}$ only. However, at a temperature of $100 \mathrm{~K}$, exit effects are important for all the molecules considered in this study with the exception of $\mathrm{Ar}$ and $\mathrm{Ne}$, which are the two smallest molecules considered in this study. In fact, at this temperature the energy barriers at the pore exit corresponding to $\mathrm{SnBr}_{4}$ and $\mathrm{CCl}_{4}$ are 92.2 and 64.8 $k_{\mathrm{B}} T$, respectively. Such large barriers would essentially prevent the molecule from escaping once it has entered the pore. It is also possible that a surface barrier may enhance the escape of a molecule from the pore. For example, in the case of $\mathrm{Ne}$, it is observed that the intracrystalline barrier is larger than the pore exit barrier at all temperatures (this can be observed from the $\mathrm{PMF}$ of $\mathrm{Ne}$ at $300 \mathrm{~K}$ ), thus leading to a larger transport

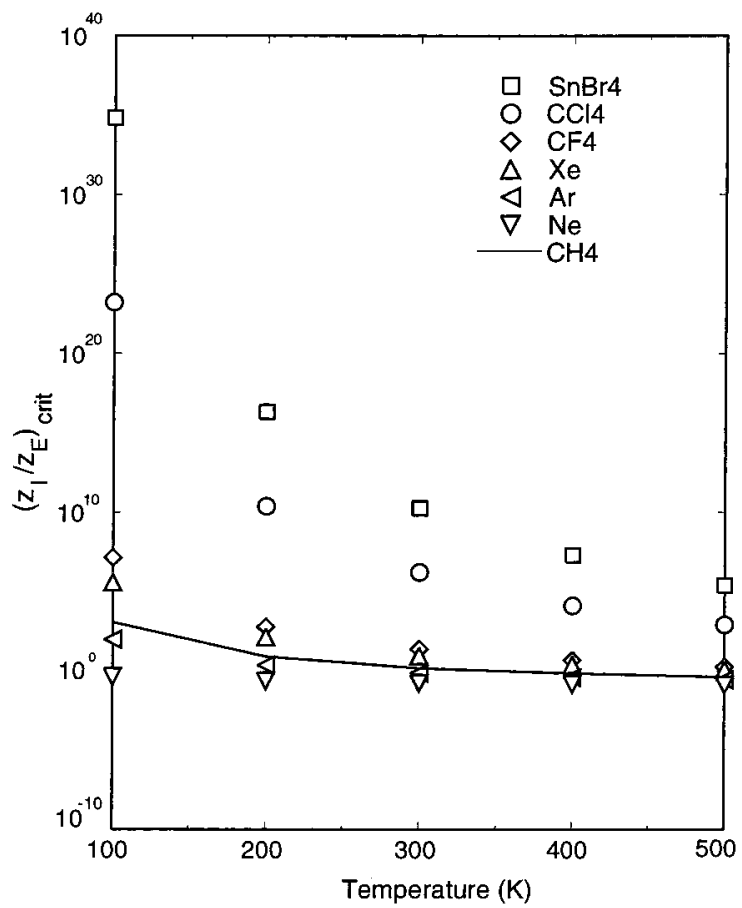

Figure 10. Predicted relative importance of the surface barrier resistance to the intracrystalline resistance, $\left(z_{\mathrm{I}} / z_{\mathrm{E}}\right)_{\text {crit }}$, using the activated transport model, at different temperatures for the various molecules considered in this study.

coefficient at the exit than in the intracrystalline region (consequently $\left(z_{\mathrm{I}} / z_{\mathrm{E}}\right)_{\text {crit }}$ is less than 1$)$. The same argument could be used to explain the fact that some of the transport coefficients obtained for methane from the DCV-GCMD simulations (Table 2) were larger at the pore exit than in the intracrystalline regions of the pore. As expected, the PMF profile in Figure 8 at $T=$ $500 \mathrm{~K}$ shows that the intracrystalline activation energy is higher than that corresponding to the pore exit.

\section{Conclusions}

In this paper, we have addressed the problem of the pore exit limiting the rate of transport of $\mathrm{CH}_{4}$ molecules across a $\mathrm{AlPO}_{4}-5$ pore. This issue is important from the industrial perspective because zeolites are used commercially for separation of gases on the basis of their relative sizes. The rate of separation is therefore dependent on the rate of transport of sorbates through these zeolite pores. Most of the literature considers the effect of natural surface barriers in these types of zeolites on the adsorption of sorbates only. In this paper we have shown that the pore exit barrier may also impact transport by hindering the desorption process.

To study this effect, we first conducted DCV-GCMD simulations to evaluate the transport coefficients in the intracrystalline 
region of the pore and at the pore exit. We observed that the relative importance of the barrier decreases with temperature. Interestingly, the surface barrier effects are much more severe at low sorbate loadings where the escape of the sorbate molecule is unaffected by the presence of other sorbate molecules. At high sorbate loadings, the local clustering of molecules causes a significant lowering of the pore exit barrier. The relative importance of the surface energy barrier has been quantified by plotting the ratio of intracrystalline pore length to the length of the exit region required for the surface barrier resistance to become equal to the intracrystalline resistance. The smallest intracrystalline length of the pore required for exit effects to be unimportant at $T=100 \mathrm{~K}$ is about $340 \AA$. Most commercial zeolite crystals are considerably larger than this, and usually operate at temperatures higher than $100 \mathrm{~K}$. Therefore, the effect of surface barrier in the case of methane in $\mathrm{AlPO}_{4}-5$ with loadings between 0.5 and 2.5 molecules/unit cell is negligible.

The simulation approach is computationally demanding, and thus not the ideal tool for studying exit effects for a wide range of species. To this end, a simple theoretical model is developed which predicts an upper bound on the relative importance of exit effects at different temperatures, and for different molecules. The dependence of the exit effect importance on the temperature is well captured by this model, though it fails at low temperatures, which is most likely due to its neglect of molecular clustering. The model also shows that exit effects become more prominent as the ratio of the sorbate to pore diameter approaches unity. It was found that, at $T=300 \mathrm{~K}$, exit effects in a $1 \mu \mathrm{m}$ $\mathrm{AlPO}_{4}-5$ crystal are significant for $\mathrm{SnBr}_{4}$ and $\mathrm{CCl}_{4}$. At a lower temperature $(T=100 \mathrm{~K})$, smaller molecules such as $\mathrm{CF}_{4}, \mathrm{Xe}$, and $\mathrm{CH}_{4}$ also start exhibiting exit effects. The activated transport model could also be used to estimate the impact of pore exit effects in other sorbate-zeolite systems.

Acknowledgment. Computational resources were provided by a grant from the Army Research Office (DAAG55-9810091). The Center of Applied Mathematics, University of Notre Dame, provided financial assistance and a graduate fellowship to G.A. E.J.M. acknowledges the National Science Foundation CAREER program (Grant CTS-9701470) for its support. H.C.C. acknowledges support from the Bayer chair fund. We also thank Michael Macedonia for some of the codes he provided.

\section{References and Notes}

(1) Bhatia, S. K.; Liu, F.; Arvind, G. Langmuir 2000, 16, 4001.

(2) Barrer, R. M. Langmuir 1987, 3, 309.

(3) Kärger, J.; Ruthven, D. Diffusion in Zeolites and Other Microporous Solids; Wiley: New York, 1991.

(4) Kärger, J.; Bülow, M.; Millward, G. R.; Thomas, J. M. Zeolites 1986, 6,146 .

(5) Kärger, J.; Pfeifer, H.; Stallmach, F.; Splinder, H. Zeolites 1990 $10,288$.

(6) Caro, J.; Bülow, M.; Kärger, J. AIChE J. 1980, 26, 1044.

(7) Bülow, M.; Struve, P.; Rees, L. V. C. Zeolites 1985, 5, 113.

(8) Kočiř́ík, M.; Struve, P.; Fiedler, K.; Bülow, M. J. Chem. Soc., Faraday Trans. 1 1988, 88, 3001.

(9) Ford, D. M.; Glandt, E. D. J. Membr. Sci. 1995, 107, 47.

(10) Kärger, J. Langmuir 1988, 4, 1289.

(11) Vigné-Maeder, F.; El Amrani, S.; Gélin, P. J. Catal. 1992, 134 536.

(12) Jobic, H.; Beé, M.; Caro, J.; Bülow, M.; Kärger, J. J. Chem. Soc., Faraday Trans. 1 1989, 85, 4201.

(13) Ford, D. M.; Glandt, E. D. J. Phys. Chem. 1995, 99, 11543.

(14) Webb, E. B., III; Grest, G. S. In Dynamics in Small Confining Systems IV; Drake, J. M., Grest, G. S., Klafter, J., Kopelman, R., Eds.; MRS: Warrendale, PA, 1999.

(15) Heffelfinger, G. S.; van Swol, F. J. Chem. Phys. 1994, 100, 7548.

(16) Macedonia, M. D.; Maginn, E. J. Mol. Phys. 1999, 96, 1375.

(17) Goodbody, S. J.; Watanabe, K.; MacGowan, D.; Walton, J. P. R. B.; Quirke, N. J. Chem. Soc., Faraday Trans. 1991, 87, 1951. 261.

(18) Kiselev, A. V.; Lopatkin, A. A.; Shulga, A. A. Zeolites 1985, 5,

(19) June, R. L.; Bell, A. T.; Theodorou, D. N. J. Phys. Chem. 1990 94, 8232 .

(20) Allen, M. P.; Tildesley, D. J. Computer Simulation of Liquids; Clarendon: Oxford, 1987.

(21) Sholl, D. S.; Fichthorn, K. A. Phys. Rev. Lett. 1997, 79, 3569.

(22) Kopelevich, D. I.; Chang, H.-C. Phys. Rev. Lett. 1999, 83, 1590.

(23) McQuarrie, D. A. Statistical Mechanics; Harper and Row: New York, 1976

(24) Maginn, E. J.; Bell, A. T.; Theodorou, D. N. J. Phys. Chem. 1995, 99, 2057.

(25) Hirschfelder, J. O.; Curtis, C. F.; Bird, R. B. Molecular Theory of Gases and Liquids; Wiley: New York, 1954. 